Supporting information

\title{
Ternary $\operatorname{Ag}_{2} \mathrm{Se}_{1-\mathrm{x}} \mathrm{Te}_{\mathrm{x}}:$ a near-room-temperature thermoelectric material with potentially high figure of merit
}

Jie Chen, ${ }^{\text {a }}$ Hualei Yuan, ${ }^{\text {b }}$ Yu-Ke Zhu, ${ }^{\mathrm{c}}$ Kun Zheng, ${ }^{\mathrm{b}}$ Zhen-Hua Ge, ${ }^{\mathrm{c}}$ Jun Tang, ${ }^{\mathrm{d}}$ Dali Zhou, ${ }^{\mathrm{a},{ }^{*}}$ Lei Yang, ${ }^{\text {, }}{ }^{*}$ Zhi-Gang Chen ${ }^{\mathrm{e}}$

${ }^{a}$ School of Materials Science \& Engineering, Sichuan University, Chengdu 610064, China

${ }^{b}$ Beijing Key Lab of Microstructure and Properties of Solids, Faculty of Materials and Manufacturing, Beijing University of Technology, Beijing 100124, China

c Materials Science and Engineering, Kunming University of Science and Technology, Kunming 650093, China

${ }^{d}$ Key Laboratory of Radiation Physics and Technology, Ministry of Education, Institute of Nuclear Science and Technology, Sichuan University, Chengdu 610064, China

e Centre for Future Materials, University of Southern Queensland, Springfield Central, Queensland 4300, Australia

${ }^{*}$ Corresponding author.

E-mail address: lyang1986@scu.edu.cn (L Yang), and zdl@scu.edu.cn (DL Zhou) 

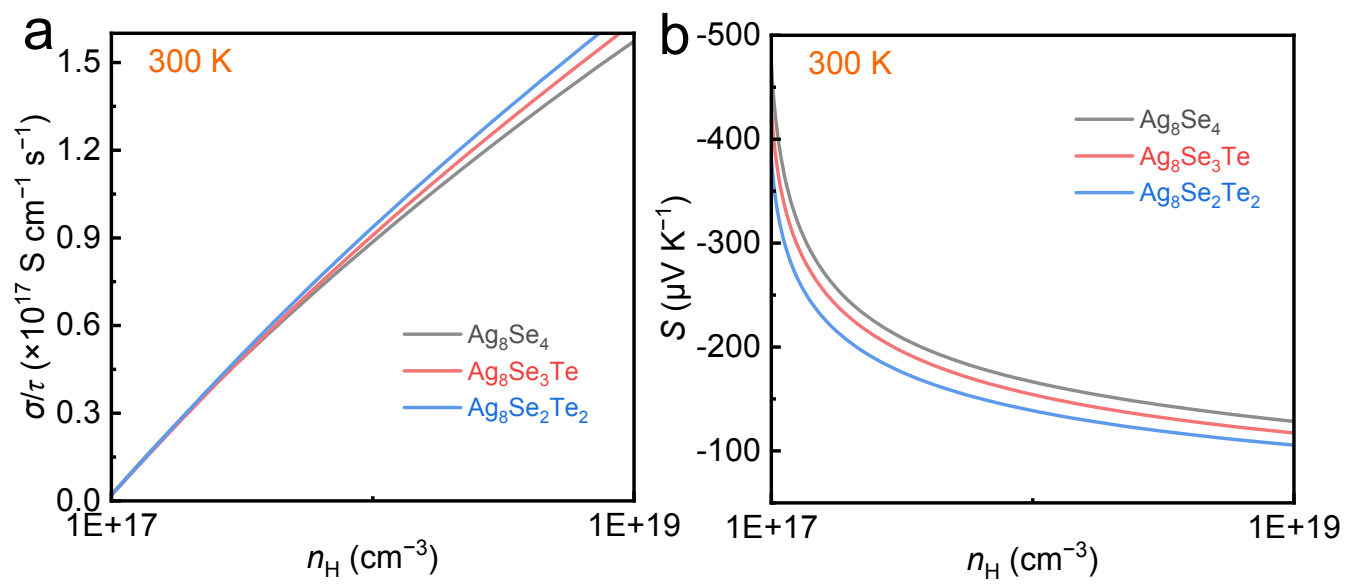

Figure S1. The theoretical transport properties as a function of $n_{\mathrm{H}}$ calculated by BoltzTraP code for $\mathrm{Ag}_{8} \mathrm{~S}_{4-\mathrm{x}} \mathrm{Te}_{\mathrm{x}}(\mathrm{x}=0,1$, and 2). (a) $\sigma / \tau$, (b) $S$. 

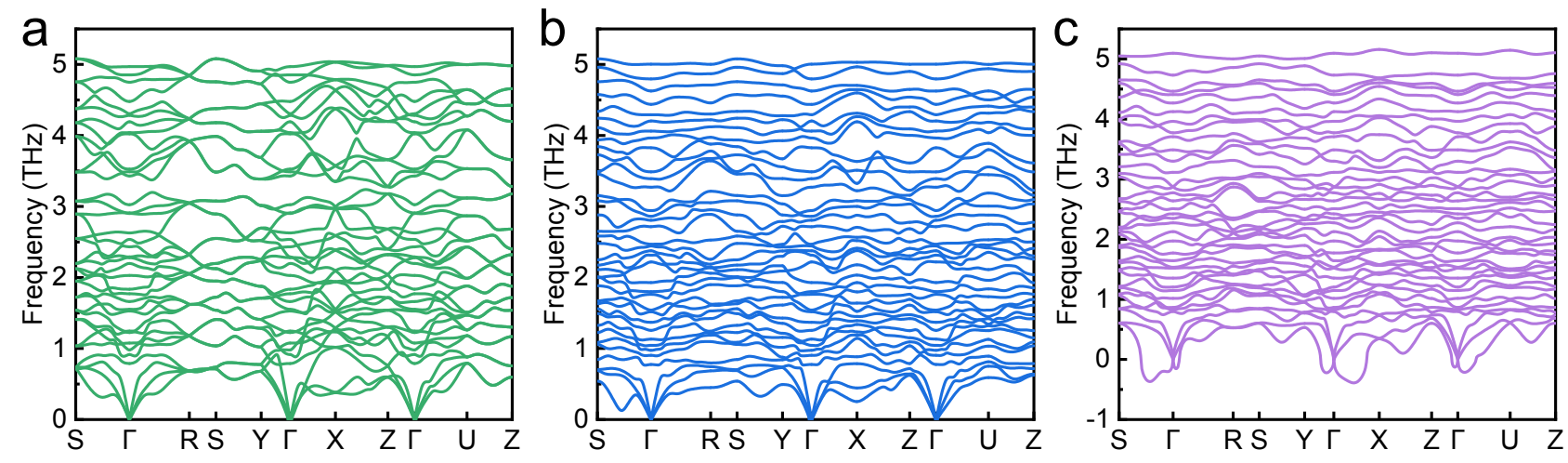

Figure S2. Phonon dispersions of (a) $\mathrm{Ag}_{64} \mathrm{Se}_{32}$, (b) $\mathrm{Ag}_{64} \mathrm{Se}_{24} \mathrm{Te}_{8}$, and (c) $\mathrm{Ag}_{64} \mathrm{Se}_{16} \mathrm{Te}_{16}$. 

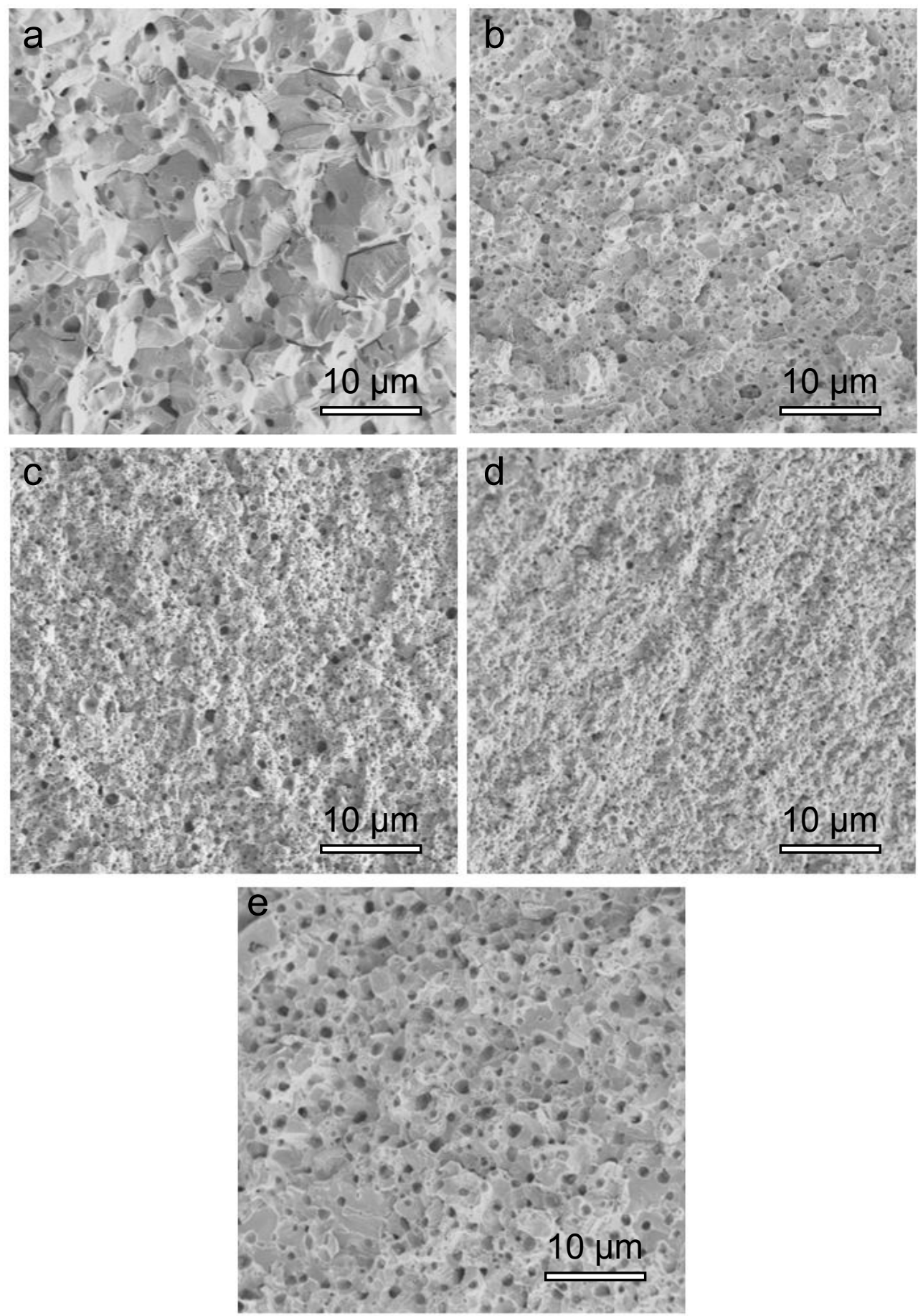

Figure S3. SEM images of the fractured sections of the samples with (a) $x=0.1$, (b) $x=0.2$, (c) $x=0.3$, (d) $\mathrm{x}=0.4$, and (e) $\mathrm{x}=0.5$. 


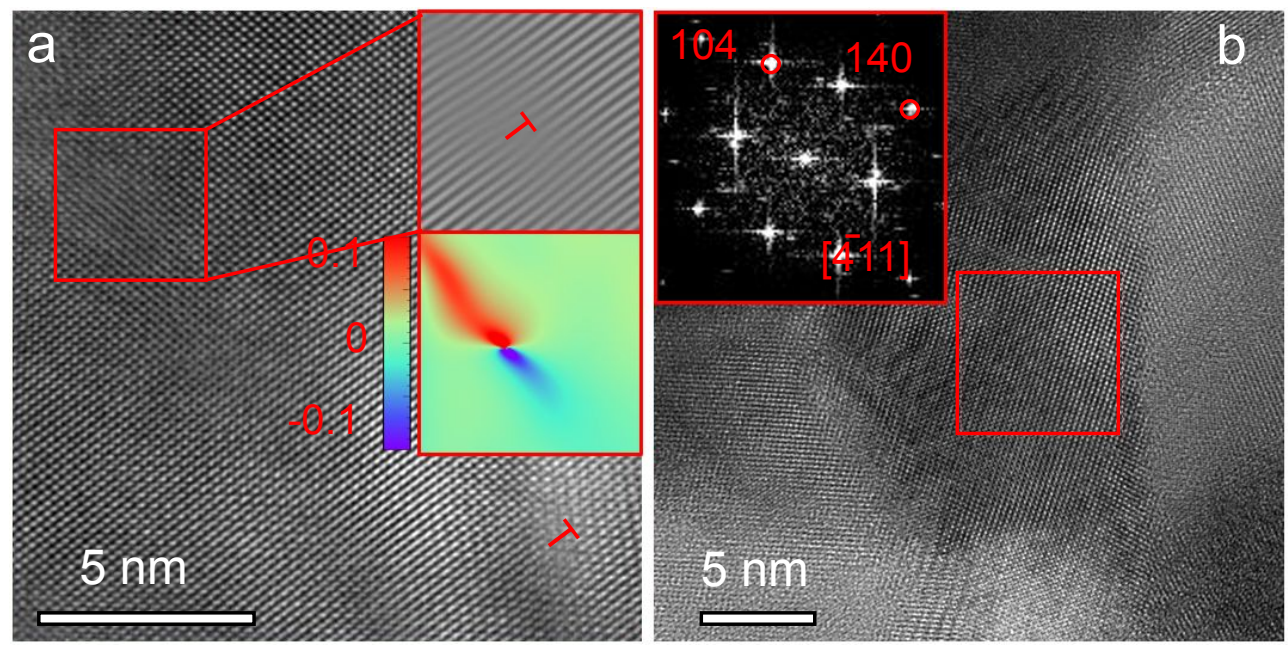

Figure S4. TEM characterized microstructures of $\mathrm{Ag}_{2} \mathrm{Se}_{0.5} \mathrm{Te}_{0.5}$. (a) HRTEM image with insets of IFFT and strain map showing the dislocation and localized strain. (b) A typical HRTEM image of a nano grain with inset of the corresponding FFT pattern. 


\section{Single parabolic band (SPB) model is presented as follow:}

Seebeck coefficient

$$
S=\frac{k_{\mathrm{B}}}{e}\left[\frac{(r+5 / 2) F_{r+3 / 2}(\eta)}{(r+3 / 2) F_{r+1 / 2}(\eta)}-\eta\right]
$$

Electrical conductivity

$$
\sigma=n_{\mathrm{H}} \mu_{\mathrm{H}} e
$$

Hall carrier concentration

$$
n_{\mathrm{H}}=4 \pi\left(\frac{2 m * k_{\mathrm{B}} T}{h^{2}}\right)^{3 / 2} \frac{F_{1 / 2}(\eta)}{r_{\mathrm{H}}}
$$

Hall factor

$$
r_{\mathrm{H}}=\frac{3}{4} \frac{F_{1 / 2}(\eta) F_{-1 / 2}(\eta)}{\left[F_{0}(\eta)\right]^{2}}
$$

Hall mobility

$$
\mu_{\mathrm{H}}=\mu_{0} \frac{(2 r+3 / 2) F_{2 r+1 / 2}(\eta)}{(r+3 / 2)^{2} F_{r+1 / 2}(\eta)}
$$

mobility parameter

$$
\mu_{0}=\frac{e \pi \hbar^{4} C_{l}}{\sqrt{2}\left(k_{\mathrm{B}} T\right)^{3 / 2} E_{\text {def }}{ }^{2}\left(m^{*}\right)^{5 / 2}}
$$

elastic constants

$$
C_{l}=\rho V_{l}^{2}
$$

Fermi integration

$$
F_{n}(\eta)=\int_{0}^{\infty} \frac{\chi^{n}}{1+\exp (\chi-\eta)} d \chi
$$

Where $k_{\mathrm{B}}$ is the Boltzmann constant, $e$ is the elementary charge, $r$ is the scattering factor $(r=-0.5$ with acoustic phonon scattering), $\eta$ is the reduced Fermi level, $m^{*}$ is the effective mass, $h$ is the Planck constant, $\hbar$ is the reduced Planck constant, $E_{\mathrm{def}}$ is the deformation potential coefficient, $v_{l}$ is the longitudinal sound velocity, $\rho$ is the density, $\chi$ is the reduced carrier energy.

The SPB model is frequently used to evaluate the thermoelectric transport properties as a function 
of $n_{\mathrm{H} \cdot}{ }^{1-2}$ The $m^{*}$ of electron was calculated by putting the measured $S$ and $n_{\mathrm{H}}$ into the equation $\mathrm{S} 1$ and S3. The $E_{\mathrm{def}}$ and $\mu_{0}$ were calculated by putting the calculated $m^{*}$ and the measured $S$ and $\mu_{\mathrm{H}}$ into the equation S1, S5 and S6. After obtaining $m^{*}$ and $\mu_{0}$, the $n_{\mathrm{H}}$-dependent thermoelectric parameters including $\sigma, S$, and $P F$ can be calculated using above equations. Then $n_{\mathrm{H}}$-dependent $z T$ can be further calculated combined with the experimental $\kappa_{1}$.

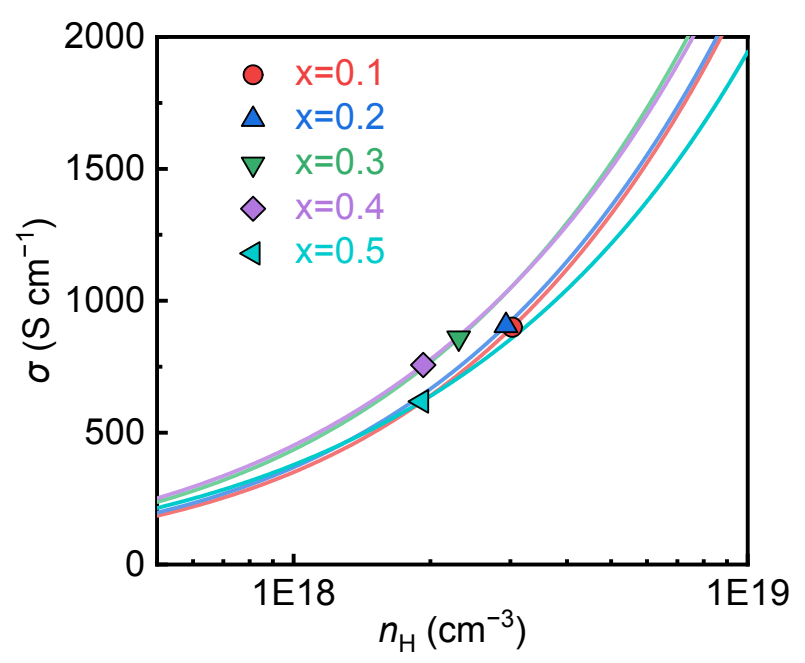

Figure S5. The $n_{\mathrm{H}}$-dependent $\sigma$ at $300 \mathrm{~K}$ calculated by SPB model. 
Electronic thermal conductivity is calculated by the Wiedemann-Franz law:

Electronic thermal conductivity

$$
\kappa_{\mathrm{e}}=L \sigma T
$$

Lorenz number

$$
L=\left(\frac{k_{\mathrm{B}}}{e}\right)^{2}\left\{\frac{(r+7 / 2) F_{r+5 / 2}(\eta)}{(r+3 / 2) F_{r+1 / 2}(\eta)}-\left[\frac{(r+5 / 2) F_{r+3 / 2}(\eta)}{(r+3 / 2) F_{r+1 / 2}(\eta)}\right]^{2}\right\}
$$

The calculated $\eta$ and $L$ are plotted in Figure S3.

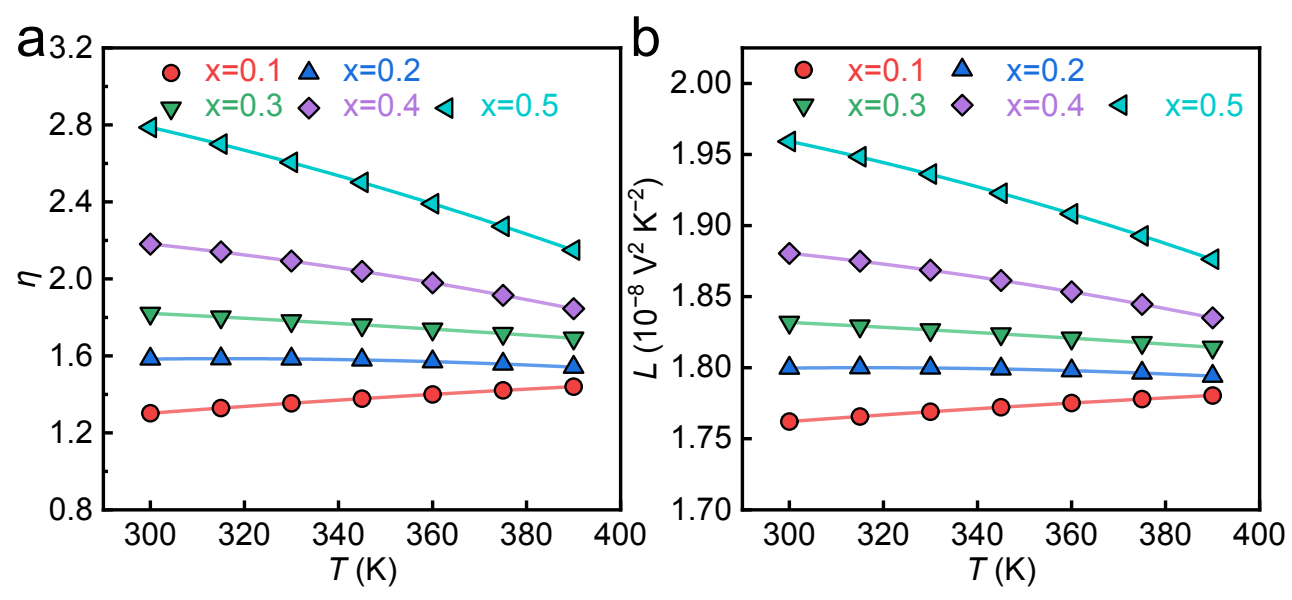

Figure S6. Temperature dependent (a) $\eta$ and (b) $L$ calculated by the Wiedemann-Franz law. 


\section{REFERENCES}

(1) Liu, W.; Chi, H.; Sun, H.; Zhang, Q.; Yin, K.; Tang, X.; Zhang, Q.; Uher, C. Advanced thermoelectrics governed by a single parabolic band: $\mathrm{Mg}_{2} \mathrm{Si}_{0.3} \mathrm{Sn}_{0.7}$, a canonical example. Phys. Chem. Chem. Phys. 2014, 16, 6893-6897.

(2) Day, T.; Drymiotis, F.; Zhang, T.; Rhodes, D.; Shi, X.; Chen, L.; Snyder, G. J. Evaluating the potential for high thermoelectric efficiency of silver selenide. J. Mater. Chem. C 2013, 1, 7568-7573. 